\title{
CONHECIMENTO DOS FATORES DE RISCO DE DOENÇAS CARDIOVASCULARES POR ESTUDANTES UNIVERSITÁRIOS: EVIDÊNCIAS CIENTÍFICAS
}

\section{KNOWLEDGE OF RISK FACTORS FOR CARDIOVASCULAR DISEASES BY UNIVERSITY STUDENTS: SCIENTIFIC EVIDENCES}

\author{
Braima Embaló $^{1} *$ Davide Carlos Joaquim $^{2} *$ Mama Saliu Culubali $^{3} *$ Virgínia Cláudia Carneiro $^{2}$ \\ Girã $^{4} *$ Daniel Freire de Sousa ${ }^{5} *$ Ana Caroline Rocha de Melo Leite ${ }^{6}$
}

\begin{abstract}
RESUMO:
Objetivo: Identificar e avaliar as evidências científicas em relação ao conhecimento de estudantes universitários sobre os fatores de risco de Doenças Cardiovasculares (DCV). Método: Trata-se de revisão integrativa, realizada em 2020, nas bases de dados MEDLINE, CINAHL, PubMed, Scopus e Web of Science. Baseado na pergunta norteadora, foi feita a busca dos artigos, utilizando-se descritores "Estudantes", "Universidades", "Conhecimento", "Doenças cardiovasculares" e "Fatores de riscos" e seus correspondentes em inglês. Resultados: Dos 17 artigos incluídos, 6 estavam disponíveis no Scopus e todos estavam em língua inglesa. Observou-se que estudantes tinham baixos níveis de conhecimento sobre os fatores de risco de DCV e não reconheciam a vulnerabilidade a elas, além de não adotarem medidas preventivas e um estilo de vida saudável. As publicações sugeriram a necessidade de profissionais de saúde em ambientes educacionais, visando aprimorar o conhecimento relacionado à saúde, incorporar práticas de estilo de vida saudáveis e desenvolver estratégias de redução dos níveis de risco de DCV. Conclusão: $O$ aprofundamento do conhecimento e o olhar crítico sobre a literatura apontaram carência na produção de estudos brasileiros sobre a temática, despertando a necessidade de condução de mais pesquisas e revisões da literatura para fortalecimento da prática baseada em evidências.
\end{abstract}

Palavras-chave: Estudantes; Universidades; Doenças Cardiovasculares; Fatores de Risco; Conhecimento.

\begin{abstract}
Objective: To identify and assess scientific evidence regarding university students' knowledge about risk factors for Cardiovascular Diseases (CVD). Method: This is an integrative review, carried out in 2020, in the MEDLINE, CINAHL, PubMed, Scopus, and Web of Science databases. Based on the guiding question, the search for articles was made, using the descriptors "Students", "Universities", "Knowledge", "Cardiovascular Diseases", and "Risk Factors" and their correspondents in English. Results: Of the 17 articles included, six were available from Scopus, and all were in English. It was observed that students have a low level of knowledge about risk factors for CVD and do not recognize their vulnerability to them, and not adopting preventive measures and a healthy lifestyle. Publications suggested the need for health professionals in educational settings to improve knowledge related to health, incorporate healthy lifestyle practices, and develop strategies to reduce CVD risk levels. Conclusion: The deepening of knowledge and a critical look at the literature showed a lack in producing Brazilian studies on the subject, arousing the need to conduct more research and literature reviews to strengthen evidence-based practice.
\end{abstract}

Keywords: Students; Universities; Cardiovascular Diseases; Risk Factors; Knowledge.

\footnotetext{
${ }^{1}$ Enfermeiro pela Universidade da Integração Internacional da Lusofonia Afro-Brasileira - UNILAB. Redenção - CE, Brasil. ORCID https://orcid.org/0000-0002-7787-1889

${ }^{2}$ Enfermeiro pela Universidade da Integração Internacional da Lusofonia Afro-Brasileira - UNILAB. Doutorando do Programa de Pós-graduação em Ciências Morfofuncionais da Universidade Federal do Ceará - UFC. Mestre pelo Programa de Pós-Graduação em Enfermagem da UNILAB. Redenção - CE, Brasil. ORCID https://orcid.org/0000-0003-0245-3110

${ }^{3}$ Enfermeiro pela Universidade da Integração Internacional da Lusofonia Afro-Brasileira - UNILAB. Redenção - CE, Brasil. ORCID https://orcid.org/0000-0002-8079-866X

${ }^{4}$ Médica Veterinária pela Universidade Estadual do Ceará - UECE. Doutora em Farmacologia pela Universidade Federal do Ceará UFC. Mestre em Ciências Veterinárias pela UECE. Docente do Departamento de Morfologia da UFC. Fortaleza, Ceará - Brasil. ORCID https://orcid.org/0000-0002-0676-8585

${ }^{5}$ Farmacêutico pela Universidade Federal do Ceará - UFC. Doutor em Biotecnologia em Saúde pela Rede Nordeste de Biotecnologia - RENORBIO. Docente dos Cursos de Enfermagem e Farmácia da Universidade da Integração Internacional da Lusofonia AfroBrasileira - UNILAB. Redenção - CE, Brasil. ORCID https://orcid.org/0000-0002-2333-5343

${ }^{6}$ Odontóloga pela Universidade Federal do Ceará - UFC. Doutora em Ciências Médicas pela UFC. Mestre em Farmacologia pela UFC. Docente dos Cursos de Enfermagem e Farmácia da Universidade da Integração Internacional da Lusofonia Afro-Brasileira UNILAB. Redenção - CE, Brasil. ORCID https://orcid.org/0000-0002-9007-7970
} 


\section{INTRODUÇÃO}

de DCV entre adultos jovens, representados

No contexto atual, as Doenças Crônicas Não especialmente pela obesidade e sedentarismo ${ }^{13}$. Transmissíveis (DCNT), como as Doenças Particularmente, o maior acometimento de faixas Cardiovasculares (DCV), diabetes, câncer e doenças etárias mais jovens por DCV pode se relacionar à crônicas respiratórias ${ }^{1}$, representam um grave percepção de que essas doenças só ocorrem em problema para a saúde pública mundial, como indivíduos de maior idade ${ }^{14,15}$. Contribuem ainda consequência do elevado número de óbitos e para essa realidade as mudanças alimentares incapacitação ${ }^{2}$ observado entre os países vivenciadas por esse público como consequência do desenvolvidos e subdesenvolvidos ${ }^{3}$. De fato, as processo de urbanização e fácil acesso a alimentos DCNT são responsáveis pela morte de 41 milhões processados ${ }^{16}$.

de pessoas por ano, quantitativo correspondente a Diante desse novo cenário, estudantes $71 \%$ do total de óbitos ${ }^{4}$. Em termos universitários se destacam como um grupo etiopatogênicos, elas resultam da atuação de fatores vulnerável a DCV, especialmente se considerado o genéticos, fisiológicos, ambientais e estilo de vida ${ }^{4}$. seu comportamento sedentário ${ }^{17,18}$, consumo de

Definidas como um grupo de desordens que álcool e cigarro ${ }^{19}$, estresse ${ }^{20}$ e alteração do hábito acometem o coração e a circulação arterial e alimentar ${ }^{21}$. Corroborando com essa suposição, $\operatorname{venosa}^{5,6}$, as quais incluem a doença arterial estudos relatam a presença de fatores de risco de coronariana e cerebrovascular e aterosclerose DCV entre estudantes de diferentes cursos, como aórtica $^{7}$, as DCV representam a principal causa de Medicina $^{22}$, Enfermagem ${ }^{23}$, Nutrição e morte no mundo ${ }^{8}$. Realmente, essa condição é Fisioterapia ${ }^{24}$.

responsável por $30 \%$ dos 50 milhões de óbitos Nesse sentido, a literatura ressalta que o registrados mundialmente nas últimas décadas ${ }^{9}$. Sua ingresso na Universidade favorece o contato e etiologia, embora envolva fatores tidos como não desenvolvimento de fatores de risco de DCV pelos modificáveis, como sexo, idade, história familiar e estudantes, em particular aqueles que se veem raça, compreende elementos modificáveis ou obrigados a se distanciar da família e assumir a evitáveis, como sedentarismo, obesidade, responsabilidade pela sua moradia, alimentação e Hipertensão Arterial Sistêmica (HAS), Diabetes condições financeiras ${ }^{23,25}$. Com o decorrer do curso, Mellitus (DM), dislipidemia, tabagismo, dieta somam-se outros fatores, como a carga horária inadequada e ingestão de álcool ${ }^{10,11}$. extensa em sala de aula e estágio, adoção de

Embora acometa principalmente adultos consumo de alimentos industrializados pela acima de 60 anos $^{6}$, além da ocorrência em escassez de tempo e dinheiro, limitado acesso a indivíduos com idade inferior a 40 anos, geralmente alimentos saudáveis no ambiente universitário e associada a condições genéticas ${ }^{12}$, a literatura reduzida disponibilidade e tempo para a prática de mostra o aumento da prevalência de fatores de risco exercício físico ${ }^{23,25,26}$. 
Apesar da presença desses fatores de risco interpretação dos resultados; relato da revisão e entre os universitários, estudos revelam um déficit síntese do conhecimento evidenciado nas pesquisas. no conhecimento desses fatores entre esses Para a formulação da pergunta norteadora, foi estudantes, o que pode interferir no seu estado de utilizada a estratégia PICO, a qual representa um saúde. Nesse sentido, segundo os autores ${ }^{27}$, a acrônimo em inglês que significa "paciente, avaliação dos níveis de conhecimento e intervenção, comparação e resultados”. Esses identificação desses fatores de risco são quatros componentes são fundamentais para a fundamentais para a promoção da saúde no contexto questão da pesquisa e construção da pergunta das DCV, principalmente para a comunidade norteadora, particularmente quando estuda um tema acadêmica que, muitas vezes, é referência para a muito específico ${ }^{29}$.

sociedade.

Com base nessa estratégia, a presente revisão

Assim, considerando-se o acima exposto, esse considerou "P" estudantes universitários, "I" e "C" estudo teve como objetivo identificar e avaliar as não foram aplicados e "O" conhecimento sobre os evidências científicas em relação ao conhecimento fatores de riscos de doenças cardiovasculares. A fim de estudantes universitários sobre os fatores de risco de responder ao objetivo do estudo, foi definida a de DCV.

\section{MÉTODOS} seguinte pergunta norteadora: "O que os estudantes universitários sabem sobre os fatores de risco de

Trata-se de uma revisão integrativa da artigos, foram utilizados os descritores controlados literatura, a qual objetiva identificar, descrever, em português "Estudantes", "Universidades", reunir e sintetizar resultados de estudos. Quando "Conhecimento", "Doenças cardiovasculares" e bem elaborada, possibilita a integração de pesquisas "Fatores de riscos", indexados nos descritores em referentes a um determinado assunto, propiciando o Ciências da Saúde (DeCS), e seus respectivos desenvolvimento e direcionamento de outras correspondentes em inglês "Students", investigações, auxiliando na tomada de decisão e "Universities", "Knowledge", "Cardiovascular permitindo a incorporação da evidência na prática diseases" e "Risk factors". Todas as palavras foram clínica $^{28}$. combinadas conjuntamente e associadas por meio

Com base nos autores ${ }^{29}$, essa revisão foi do operador booleano "AND". elaborada em seis etapas, a saber: identificação do

A busca e análise dos artigos foram realizadas tema e objetivos da revisão integrativa; formulação durante os meses de fevereiro e março de 2020, por da questão norteadora; busca na literatura e seleção meio da consulta do Portal de Periódico da criteriosa das pesquisas; categorização dos estudos Coordenação de Aperfeiçoamento de Pessoal de encontrados e análise das publicações incluídas; Nível Superior (CAPES)/Ministério da Educação (MEC), usando as seguintes bases de dados: 
Medical Literature Analysis and Retrieval System verificados o objetivo, tipo de estudo e nível de Online (MEDLINE); Cumulative Index to Nursing evidência e, posteriormente, os resultados e and Allied Health Literature (CINAHL); National conclusão.

Library of Medicine (PubMed); Scopus - base A análise da classificação das evidências dos multidisciplinar e Web of Science. artigos foi fundamentada na proposta de Stillwell et

Foram incluídos na revisão artigos na íntegra, al. ${ }^{32}$. Esses autores categorizam as evidências de disponíveis gratuitamente, publicados nos últimos pesquisa em sete níveis, nos quais o primeiro 10 anos (2010 a 2020) (esse recorte temporal se corresponde a melhor evidência externa, baseou na possibilidade de incluir um maior apresentando informações mais confiáveis, e o número de estudos que abordassem a temática), nos último compreende informações com um menor idiomas português, inglês e espanhol. Foram grau de confiabilidade.

excluídos artigos repetidos, editoriais, cartas ao editor, teses, dissertações, artigos de revisão, RESULTADOS relatórios e artigos não relacionados à temática ou

A partir da estratégia de busca, foram não relevantes para essa revisão. encontrados 262 trabalhos, dos quais 191 tinham

Após a coleta, os dados foram organizados sido publicados nos últimos 10 anos. Desses, 93 com base no instrumento adaptado de $\mathrm{Ursi}^{30}$, estavam nos idiomas inglês, espanhol ou português constituído por um modelo específico de CheckList, e, dentre eles, 71 tinham disponibilidade na íntegra. dividido em nove domínios, cujo objetivo é facilitar Após a leitura do título e resumo, das 71 a descrição e publicação dos dados presentes nos publicações, foram excluídas 42 por não se $\operatorname{artigos}^{31}$. Esses foram primeiramente organizados relacionaram ao objetivo da revisão. Dos 29 artigos de acordo com: título do artigo; autores, periódico e lidos na íntegra, 9 foram excluídos por duplicidade área de publicação; local onde a pesquisa foi e 3 por não responderem os critérios de inclusão. conduzida e ano em que foi publicado e base de Assim, compuseram a revisão 17 publicações dados em que foi indexado. Na sequência, foram (Figura 1). 
Figura 1 - Fluxograma de identificação do processo de seleção dos estudos para compor a revisão integrativa. Redenção - CE, Brasil, 2020

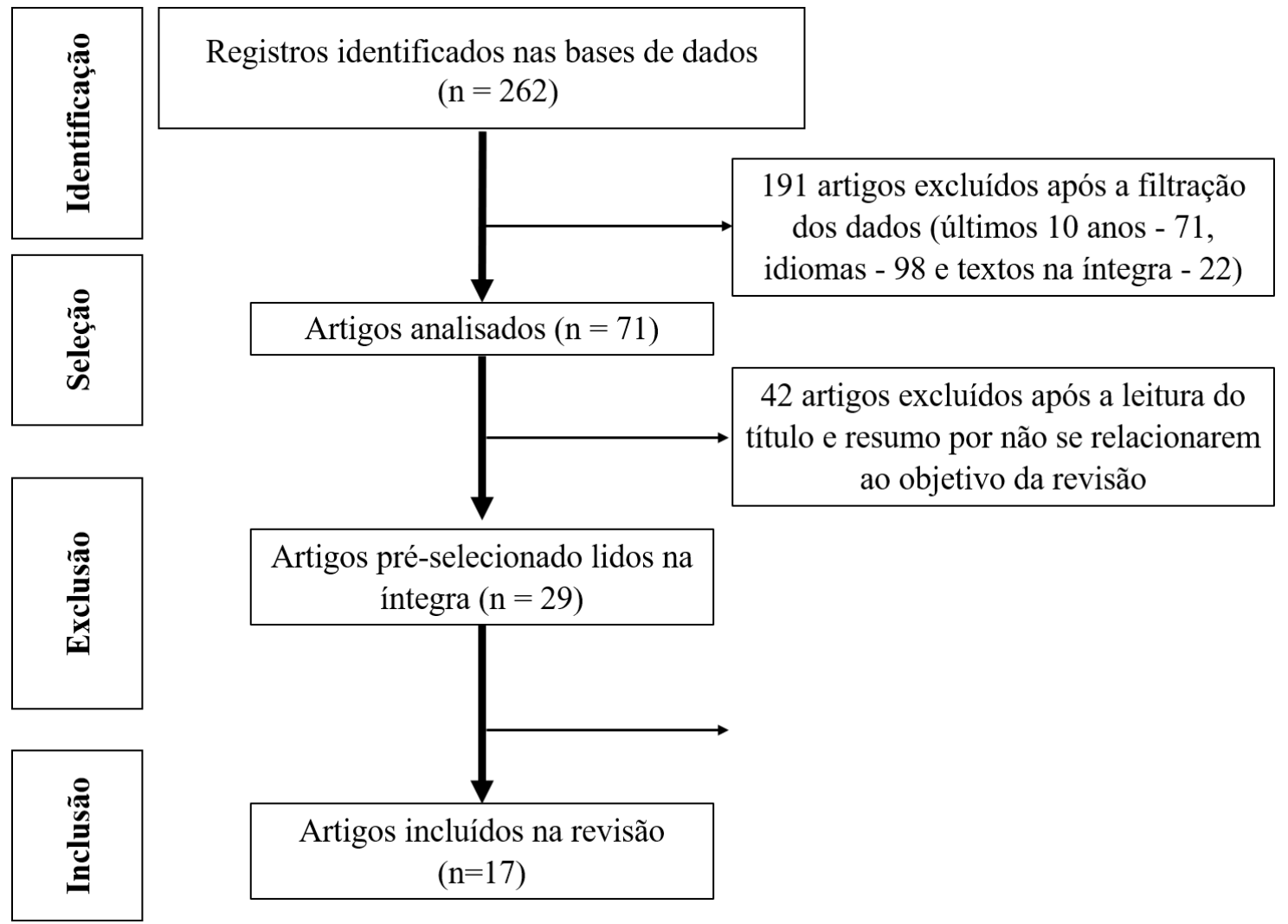

Fonte: Os autores

Do total de artigos incluídos, 5 estavam relação ao local de realização dos estudos, 3 foram disponíveis no Scopus e todos estavam em língua conduzidos na Turquia, número igual ao constatado inglesa. Quanto ao ano de publicação, 3 foram nos Estados Unidos da América. Sobre a área de publicados em 2012, quantitativo igual ao publicação, 11 estudos foram desenvolvidos na área observado nos anos de 2015, 2017 e 2019. Em da Medicina (Tabela 1).

Tabela 1 - Caracterização das publicações incluídas na revisão, de acordo com com o título, autores, ano, periódico, área, país e base de dados. Redenção - CE, Brasil, 2020.

\begin{tabular}{|c|c|c|c|c|c|}
\hline $\mathbf{N}^{\mathbf{o}}$ & Título do Artigo & Autor e Ano & Periódico e Área & País & $\begin{array}{l}\text { Base de } \\
\text { Dados }\end{array}$ \\
\hline 01 & $\begin{array}{l}\text { Knowledge, attitude, and } \\
\text { practice on salt and } \\
\text { assessment of dietary salt } \\
\text { and fat intake among } \\
\text { University of Sharjah } \\
\text { Students }\end{array}$ & $\begin{array}{l}\text { Ismael et al. } \\
2019\end{array}$ & $\begin{array}{c}\text { Journal Nutrients } \\
\text { Nutrição }\end{array}$ & $\begin{array}{l}\text { Emirados } \\
\text { Árabes } \\
\text { Unidos }\end{array}$ & Scopus \\
\hline $\mathbf{0 2}$ & $\begin{array}{l}\text { Cardiovascular risk factors } \\
\text { among lower Silesian }\end{array}$ & $\begin{array}{l}\text { Kalka et al. } \\
2016\end{array}$ & $\begin{array}{l}\text { Adv Clin Exp } \\
\text { Med }\end{array}$ & $\begin{array}{c}\text { Sul e } \\
\text { Sudoeste da }\end{array}$ & Scopus \\
\hline
\end{tabular}




\begin{tabular}{|c|c|c|c|c|c|}
\hline & $\begin{array}{l}\text { students of the Faculty of } \\
\text { Medicine: knowledge and } \\
\text { distribution }\end{array}$ & & Medicina & Polônia & \\
\hline 03 & $\begin{array}{l}\text { Knowledge, attitude, and } \\
\text { practice of Malaysian Public } \\
\text { University students on risk } \\
\text { factors for cardiovascular } \\
\text { diseases }\end{array}$ & $\begin{array}{l}\text { Ibrahim et al. } \\
2016\end{array}$ & $\begin{array}{c}\text { Journal of } \\
\text { Applied } \\
\text { Pharmaceutical } \\
\text { Science } \\
\text { Medicina }\end{array}$ & Malásia & Scopus \\
\hline 04 & $\begin{array}{l}\text { Prevalence of obesity and } \\
\text { hypertension among } \\
\text { University students' and } \\
\text { their knowledge and attitude } \\
\text { towards risk factors of } \\
\text { Cardiovascular Disease } \\
\text { (CVD) in Jeddah, Saudi } \\
\text { Arabia }\end{array}$ & $\begin{array}{l}\text { Baig et al. } \\
2015\end{array}$ & $\begin{array}{c}\text { Pak J Med Sci } \\
\text { Medicina }\end{array}$ & $\begin{array}{l}\text { Arábia } \\
\text { Saudita }\end{array}$ & Scopus \\
\hline 05 & $\begin{array}{lr}\text { Diabetes } & \text { Mellitus-related } \\
\text { knowledge } & \text { among } \\
\text { University } & \text { students in } \\
\text { Ajman, } & \text { United Arab } \\
\text { Emirates } & \\
\end{array}$ & $\begin{array}{l}\text { Khan et al. } \\
\quad 2012\end{array}$ & $\begin{array}{l}\text { SQU Medical } \\
\text { Journal } \\
\text { Medicina }\end{array}$ & $\begin{array}{l}\text { Emirados } \\
\text { Árabes } \\
\text { Unidos }\end{array}$ & Scopus \\
\hline 06 & $\begin{array}{l}\text { Awareness } \\
\text { cardiovascular risk factors } \\
\text { among University students } \\
\text { in Turkey }\end{array}$ & $\begin{array}{c}\text { Güneş et al. } \\
2019\end{array}$ & $\begin{array}{l}\text { Primary Health } \\
\text { Care Research \& } \\
\text { Development } \\
\text { Medicina }\end{array}$ & Turquia & PubMed \\
\hline 07 & $\begin{array}{l}\text { Prevalence, risk awareness } \\
\text { and health beliefs of } \\
\text { behavioral risk factors for } \\
\text { cardiovascular disease } \\
\text { among University students } \\
\text { in nine ASEAN countries }\end{array}$ & $\begin{array}{c}\text { Peltzer et al. } \\
2018\end{array}$ & $\begin{array}{l}\text { BMC Public } \\
\text { Health } \\
\text { Farmácia }\end{array}$ & $\begin{array}{l}\text { Nove países } \\
\text { da ASEAN* }\end{array}$ & PubMed \\
\hline 08 & $\begin{array}{l}\text { Assessment of knowledge } \\
\text { of critical cardiovascular } \\
\text { risk indicators among } \\
\text { college students: does stage } \\
\text { of education matter? }\end{array}$ & $\begin{array}{c}\text { Sarpong et al. } \\
2017\end{array}$ & $\begin{array}{l}\text { Int. J. Environ. } \\
\text { Res. Public } \\
\text { Health } \\
\text { Enfermagem }\end{array}$ & $\begin{array}{l}\text { Estados } \\
\text { Unidos da } \\
\text { América }\end{array}$ & PubMed \\
\hline 09 & $\begin{array}{l}\text { The perception and } \\
\text { knowledge } \\
\text { cardiovascular risk factors } \\
\text { among Medical students }\end{array}$ & $\begin{array}{c}\text { Reiner et al. } \\
2012\end{array}$ & $\begin{array}{c}\text { Meoica'l } \\
\text { Education- Croat } \\
\text { Med J. } \\
\text { Medicina } \\
\end{array}$ & Croácia & Medline \\
\hline 10 & $\begin{array}{l}\text { Cardiovascular health risk } \\
\text { behavior among Medical } \\
\text { students in a teaching } \\
\text { hospital }\end{array}$ & $\begin{array}{c}\text { Giri et al. } \\
2012\end{array}$ & $\begin{array}{l}\text { J Nepal Health } \\
\text { Res Counc } \\
\text { Medicina } \\
\end{array}$ & Nepal & Medline \\
\hline 11 & $\begin{array}{l}\text { Awareness and knowledge } \\
\text { of cardiovascular disease } \\
\text { risk factors among Medical }\end{array}$ & $\begin{array}{c}\frac{\text { Maksimović }}{\text { et al. }} \\
2017\end{array}$ & $\begin{array}{c}\text { Wien Klin } \\
\text { Wochenschr }\end{array}$ & Sérvia & Medline \\
\hline
\end{tabular}




\begin{tabular}{|c|c|c|c|c|c|}
\hline & students & & Medicina & & \\
\hline 12 & $\begin{array}{l}\text { An } \\
\text { cardiovascular } \\
\text { education progriential } \\
\text { African Amealth } \\
\text { students }\end{array}$ & $\begin{array}{l}\text { Holland et al. } \\
2014\end{array}$ & $\begin{array}{l}\text { The ABNF } \\
\text { Journal } \\
\text { Medicina }\end{array}$ & $\begin{array}{l}\text { Estados } \\
\text { Unidos da } \\
\text { América }\end{array}$ & $\begin{array}{l}\text { Web of } \\
\text { Science }\end{array}$ \\
\hline 13 & $\begin{array}{l}\text { Cardiovascular disease risk } \\
\text { factors and knowledge level } \\
\text { in Nursing students }\end{array}$ & $\begin{array}{l}\text { Kaya et al. } \\
2019\end{array}$ & $\begin{array}{l}\text { Clin Exp Health } \\
\text { Sci } \\
\text { Enfermagem }\end{array}$ & Turquia & $\begin{array}{l}\text { Web of } \\
\text { Science }\end{array}$ \\
\hline 14 & $\begin{array}{l}\text { Factors predicting nutrition } \\
\text { and physical activity } \\
\text { behaviors due to } \\
\text { cardiovascular disease in } \\
\text { Tehran University students: } \\
\text { application of health belief } \\
\text { model }\end{array}$ & $\begin{array}{c}\text { Najarkolaei et } \\
\text { al. } \\
2015\end{array}$ & $\begin{array}{c}\text { Iran Red Crescent } \\
\text { Med J } \\
\text { Medicina }\end{array}$ & Teerã & $\begin{array}{l}\text { Web of } \\
\text { Science }\end{array}$ \\
\hline 15 & $\begin{array}{l}\text { Knowledge, attitude and } \\
\text { behaviour regarding dietary } \\
\text { salt intake among Medical } \\
\text { students in Angola }\end{array}$ & $\begin{array}{l}\text { Magalhães et } \\
\text { al. } \\
2015\end{array}$ & $\begin{array}{c}\text { Cardiovascular } \\
\text { Journal of Africa } \\
\text { Medicina }\end{array}$ & Angola & $\begin{array}{l}\text { Web of } \\
\text { Science }\end{array}$ \\
\hline 16 & $\begin{array}{l}\text { University students' } \\
\text { knowledge levels about } \\
\text { cardiovascular risk factors } \\
\text { and assessment of their } \\
\text { health behaviours in Turkey }\end{array}$ & $\begin{array}{c}\text { Kes et al. } \\
2018\end{array}$ & $\begin{array}{c}\text { Journal of } \\
\text { American College } \\
\text { Health } \\
\text { Enfermagem }\end{array}$ & Turquia & CINAHL \\
\hline 17 & $\begin{array}{l}\text { Cardiovascular risk factors } \\
\text { among college students: } \\
\text { knowledge, perception, and } \\
\text { risk assessment }\end{array}$ & $\begin{array}{c}\text { Tran et al. } \\
2017\end{array}$ & $\begin{array}{c}\text { International } \\
\text { Journal of Caring } \\
\text { Sciences } \\
\text { Enfermagem } \\
\end{array}$ & $\begin{array}{l}\text { Estados } \\
\text { Unidos da } \\
\text { América }\end{array}$ & CINAHL \\
\hline
\end{tabular}

*ASEAN - Associação das Nações do Sudeste Asiático.

Fonte: Os autores

Quanto aos objetivos dos estudos, eles público; - avaliar o impacto da educação médica no envolveram principalmente avaliar os conhecimento e reconhecimento da importância da conhecimentos, atitudes e práticas em relação aos implementação de medidas preventivas contra fatores de risco de DCV por parte dos estudantes DCV, segundo a percepção dos estudantes de universitários. Outros objetivos mencionados Medicina; - conhecer os resultados da avaliação de corresponderam a: - estudar a prevalência de risco de DCV entre universitários; - identificar os obesidade e hipertensão entre os universitários; - preditores importantes de nutrição e atividade física investigar os conhecimentos e práticas relacionados em relação às DCV nesses estudantes (Tabela 2).

ao DM desses estudantes; - determinar a No que se refere ao desenho metodológico, 16 prevalência de fatores de risco de DCV nesse artigos eram estudos descritivos e, sobre o nível de 
evidência, todos as publicações apresentaram nível VI.

Tabela 2 - Caracterização das publicações incluídas nessa revisão, de acordo com o objetivo, tipo de estudo e nível de evidência. Redenção - CE, Brasil, 2020.

\begin{tabular}{|c|c|c|c|}
\hline $\mathbf{N}^{\mathbf{o}}$ & Objetivo & Tipo de Estudo & Nível de Evidência \\
\hline 01 & $\begin{array}{l}\text { Investigar os conhecimentos, atitudes e práticas } \\
\text { relacionados à presença de sal na dieta de } \\
\text { estudantes da } \operatorname{UOS}^{\mathrm{a}} \text { e avaliar a ingestão alimentar } \\
\text { de gordura total, saturada e trans, colesterol e sódio }\end{array}$ & Descritivo & VI \\
\hline 02 & $\begin{array}{l}\text { Avaliar o conhecimento dos estudantes de } \\
\text { Medicina sobre os fatores de risco de } \mathrm{DCV}^{\mathrm{b}} \text { e sua } \\
\text { prevalência na população estudada }\end{array}$ & Descritivo & VI \\
\hline 03 & $\begin{array}{l}\text { Medir e avaliar os conhecimentos, atitudes e } \\
\text { práticas de estudantes universitários em relação aos } \\
\text { fatores de risco de } \mathrm{DCV}^{\mathrm{b}}\end{array}$ & Descritivo & VI \\
\hline 04 & $\begin{array}{l}\text { Investigar a prevalência de obesidade e hipertensão } \\
\text { entre estudantes universitários e seus } \\
\text { conhecimentos e atitudes em relação aos fatores de } \\
\text { risco de } \mathrm{DCV}^{\mathrm{b}}\end{array}$ & Descritivo & VI \\
\hline 05 & $\begin{array}{l}\text { Avaliar os conhecimentos e práticas relacionados } \\
\text { ao } \mathrm{DM}^{\mathrm{c}} \text { de estudantes universitários, incluindo as } \\
\text { diferenças em relação ao gênero e histórico } \\
\text { familiar }\end{array}$ & Descritivo & VI \\
\hline 06 & $\begin{array}{l}\text { Determinar o nível de conscientização de } \\
\text { estudantes universitários, especialmente calouros, } \\
\text { em termos de fatores de risco de } \mathrm{DCV}^{\mathrm{b}} \text { (elevado } \\
\text { colesterol, estresse, hipertensão, tabagismo, } \\
\text { obesidade, diabetes, inatividade física, história } \\
\text { familiar de DCV }{ }^{\mathrm{b}} \text {, dieta não saudável, exposição ao } \\
\text { tabagismo passivo e baixo nível socioeconômico) }\end{array}$ & Descritivo & VI \\
\hline 07 & $\begin{array}{l}\text { Investigar a prevalência, consciência e crenças de } \\
\text { saúde em relação aos fatores de risco de } \operatorname{DCV}^{\mathrm{b}} \text { de } \\
\text { universitários em nove países da ASEAN }\end{array}$ & Descritivo & VI \\
\hline 08 & $\begin{array}{l}\text { Estimar e comparar o nível de conscientização de } \\
\text { estudantes do } 1^{\circ} \text { ano profissional do curso de } \\
\text { Farmácia com universitários do } 2^{\circ} \text { semestre do } \\
\text { referido curso sobre seus números (colesterol, } \\
\text { pressão arterial, glicose e IMC }{ }^{\mathrm{e}} \text { ) }\end{array}$ & Descritivo & VI \\
\hline 09 & $\begin{array}{l}\text { Avaliar as percepções, conhecimentos e } \\
\text { conscientização em relação aos fatores de risco de } \\
\text { DCV }^{\mathrm{b}} \text { entre estudantes do curso de Medicina }\end{array}$ & Descritivo & VI \\
\hline 10 & $\begin{array}{l}\text { Avaliar o comportamento em saúde e as } \\
\text { percepções de estudantes de medicina em relação a } \\
\text { doenças cardiovasculares }\end{array}$ & Descritivo & VI \\
\hline 11 & $\begin{array}{l}\text { Avaliar os conhecimentos e atitudes de estudantes } \\
\text { de Medicina com relação aos fatores de risco de } \\
\text { DCV e avaliar o impacto da educação médica no } \\
\text { conhecimento e reconhecimento da importância da } \\
\text { implementação de medidas preventivas dessas } \\
\text { doenças para esses estudantes }\end{array}$ & Descritivo & VI \\
\hline
\end{tabular}




\begin{tabular}{|l|l|c|c|}
\hline 12 & $\begin{array}{l}\text { Investigar a aplicação de um teste culturalmente } \\
\text { específico e de um currículo desenvolvido para } \\
\text { universitários afro-americanos para aumentar a } \\
\text { conscientização sobre o risco de DCV }\end{array}$ & $\begin{array}{c}\text { Descritivo e } \\
\text { Misto }\end{array}$ & VI \\
\hline $\mathbf{1 3}$ & $\begin{array}{l}\text { Determinar o conhecimento dos fatores de risco de } \\
\text { DCV e a avaliação de risco entre os estudantes de } \\
\text { Enfermagem }\end{array}$ & Descritivo \\
\hline $\mathbf{1 4}$ & $\begin{array}{l}\text { Identificar preditores importantes de nutrição e } \\
\text { atividade física em relação às DCV de estudantes } \\
\text { universitários de Teerã }\end{array}$ & Descritivo & VI \\
\hline $\mathbf{1 5}$ & $\begin{array}{l}\text { Determinar a ingestão de sal e avaliar os } \\
\text { conhecimentos, atitudes e comportamentos em } \\
\text { relação a essa ingestão entre estudantes de } \\
\text { Medicina }\end{array}$ & Descritivo \\
\hline $\mathbf{1 6}$ & $\begin{array}{l}\text { Avaliar os níveis de conhecimento de estudantes } \\
\text { universitários sobre fatores de risco de DCV e } \\
\text { seus comportamentos de saúde }\end{array}$ & Descritivo \\
\hline $\mathbf{1 7}$ & $\begin{array}{l}\text { Avaliar os conhecimentos e percepções dos } \\
\text { estudantes universitários sobre fatores de risco de } \\
\text { DCV }{ }^{b} \text { e identificar esses riscos }\end{array}$ & Descritivo & VI \\
\hline
\end{tabular}

${ }^{\mathrm{a}}$ Universidade de Sharjah (UOS); ${ }^{\mathrm{b}}$ Doenças Cardiovasculares; ${ }^{\mathrm{c} D i a b e t e s ~ M e l l i t u s ; ~}{ }^{\mathrm{d} A s s o c i a c ̧ a ̃ o ~ d a s ~}$ Nações do Sudeste Asiático; 'Índice de Massa Corporal.

Fonte: Os autores

No que diz respeito aos resultados observados de desenvolver essas doenças, não adotavam nos artigos, eles ressaltaram que, apesar das medidas preventivas e não tinham uma prática de diferenças entre os universitários de distintas estilo de vida saudável. Outros autores mostraram faculdades e tempo de curso, em geral, os ainda baixo nível de conhecimento sobre os estudantes apresentaram baixos níveis de indicadores de saúde entre os universitários, conhecimento sobre os fatores de risco de DCV. percepção de que o curso de Medicina era Dentre esses fatores, foram citados inatividade prejudicial à saúde e diferenças entre os sexos física, dieta rica em gordura, tabagismo, obesidade, quanto às atitudes e práticas em saúde.

fatores genéticos, hipertensão e DM tipo 2. Os $\quad$ Com relação à conclusão, os artigos, em estudos apontaram ainda a presença de fatores de geral, concluíram que os estudantes universitários risco entre os estudantes, como sobrepeso, tinham um nível insuficiente de consciência e obesidade, hipertensão, excesso de ingestão de conhecimento sobre os fatores de risco de DCV, gorduras e sódio, falta de atividade física, ingestão bem como elevada prevalência desses fatores entre de álcool e tabagismo (Tabela 3).

eles. Os estudos sugeriram ainda a necessidade da

Algumas pesquisas constaram ainda que, presença de profissionais de saúde em ambientes apesar da consciência dos estudantes quanto aos educacionais, a fim de aprimorar o conhecimento fatores de risco de DCV, eles não se viam em risco relacionado à saúde, incorporar práticas de estilo de 
vida saudáveis e desenvolver estratégias de redução dos níveis de risco de DCV entre os estudantes.

Tabela 3 - Caracterização das publicações incluídas nessa revisão, de acordo com os resultados e conclusão. Redenção - CE, Brasil, 2020.

\begin{tabular}{|c|c|c|}
\hline $\mathbf{N}^{\mathbf{o}}$ & Resultados & Conclusão \\
\hline 01 & $\begin{array}{l}\text { Entre os estudantes, os resultados } \\
\text { indicaram baixo escore de conhecimento } \\
\text { com relação à presença de sal na dieta, } \\
\text { assim como alta prevalência de sobrepeso } \\
(28 \%) \text {, obesidade }(14 \%) \text { e hipertensão } \\
\text { estágio } 1(31 \%) \text { e } 2(20 \%) \text {. Observou-se } \\
\text { uma elevada porcentagem de estudantes } \\
\text { que excederam a ingestão recomendada de } \\
\text { gordura total (48\%), saturada (90\%) e trans } \\
(64 \%) \text { e sódio ( } 89 \%) \text {. Todos os estudantes } \\
\text { não atenderam à ingestão adequada de } \\
\text { potássio recomendada }\end{array}$ & $\begin{array}{l}\text { Conhecimentos, atitudes e práticas relacionados ao } \\
\text { consumo de sal entre estudantes revelaram a } \\
\text { ingestão não recomendada de gordura total, } \\
\text { saturada e trans e sódio. Esse resultado destaca a } \\
\text { necessidade de desenvolver campanhas de } \\
\text { conscientização específicas em relação à cultura de } \\
\text { ingestão de sal e gordura e sua associação com a } \\
\text { saúde }\end{array}$ \\
\hline 02 & $\begin{array}{l}\text { Os estudantes identificaram corretamente } \\
\text { cinco fatores de risco de } \mathrm{DCV}^{\mathrm{a}} \text {, dos quais } \\
\text { os mais citados foram a falta de atividade } \\
\text { física e dieta rica em gordura, seguidos } \\
\text { pelo tabagismo, obesidade, fatores } \\
\text { genéticos e hipertensão. Os resultados } \\
\text { mostraram que há uma consciência } \\
\text { relativamente saudável por parte dos } \\
\text { estudantes quanto aos fatores de risco de } \\
\text { DCV } \text {. Entretanto, não se observou uma } \\
\text { correlação com um estilo de vida saudável }\end{array}$ & $\begin{array}{l}\text { O estudo revelou um nível insuficiente de } \\
\text { consciência quanto aos fatores de risco de } \mathrm{DCV}^{\mathrm{a}} \\
\text { entre estudantes de Medicina }\end{array}$ \\
\hline 03 & $\begin{array}{l}\text { Entre os universitários de diferentes } \\
\text { faculdades, foram encontradas diferenças } \\
\text { significativas em relação ao conhecimento } \\
\text { dos fatores de risco de } \mathrm{DCV}^{\mathrm{a}}\left(\mathrm{HAS}^{\mathrm{b}}, \mathrm{DM}^{\mathrm{c}} \text {, }\right. \\
\text { sedentarismo, hereditariedade, estresse, } \\
\text { tabagismo, dieta não saudável e falta de } \\
\text { exercícios físicos), assim como diferenças } \\
\text { de atitude e prática entre os sexos. Os } \\
\text { dados indicaram que a prática de estilo de } \\
\text { vida saudável não foi adequada entre os } \\
\text { participantes }\end{array}$ & $\begin{array}{l}\text { O estudo sugere que os estudantes devem aumentar } \\
\text { a prática em saúde para prevenção de } \mathrm{DCV}^{\mathrm{a}} \text {. } \\
\text { Apesar do conhecimento elevado por parte dos } \\
\text { estudantes, ele não condiz com as atitudes e práticas }\end{array}$ \\
\hline 04 & $\begin{array}{l}\text { Os resultados indicaram que } 7,5 \% \text { dos } \\
\text { participantes eram hipertensos, } 29,8 \% \\
\text { apresentavam sobrepeso, } 10,7 \% \text { eram } \\
\text { moderadamente obesos e } 7,9 \% \text { eram } \\
\text { severamente obesos. A maioria dos } \\
\text { participantes estava ciente de que um estilo } \\
\text { de vida saudável poderia impedir as DCV } \\
\text { embora não o praticassem }\end{array}$ & $\begin{array}{l}\text { Existe uma grande lacuna no conhecimento, atitude } \\
\text { e prática entre a população jovem universitária } \\
\text { saudita em relação aos fatores de risco de } \mathrm{DCV}^{\mathrm{a}}\end{array}$ \\
\hline 05 & $\begin{array}{l}\text { Dos participantes, } 25 \% \text { apresentavam } \\
\text { sobrepeso ou obesidade e apenas } 27 \% \\
\text { exercitavam-se regularmente. Sobre } \mathrm{DM}^{\mathrm{c}}\end{array}$ & $\begin{array}{l}\text { Apesar da exposição a diversas fontes de } \\
\text { informação, o nível de conhecimento dos estudantes } \\
\text { sobre o } \mathrm{DM}^{\mathrm{c}} \text { é inadequado. Recomenda-se o }\end{array}$ \\
\hline
\end{tabular}




\begin{tabular}{|c|c|c|}
\hline & $\begin{array}{l}70 \% \text { dos estudantes sabiam que a doença } \\
\text { se caracterizava pela presença de altos } \\
\text { níveis de açúcar séricos e que o histórico } \\
\text { familiar era um importante fator de risco. } \\
\text { Mais da metade dos pesquisados vinculou } \\
\text { a obesidade e inatividade física a fatores de } \\
\text { risco para DM }{ }^{\text {c }} \text {. }\end{array}$ & $\begin{array}{l}\text { engajamento de profissionais de saúde em } \\
\text { ambientes educacionais, a fim de aprimorar o } \\
\text { conhecimento relacionado à saúde e incorporar } \\
\text { práticas de estilo de vida saudáveis entre os } \\
\text { estudantes }\end{array}$ \\
\hline 06 & $\begin{array}{l}\text { O estudo revelou que os estudantes são } \\
\text { menos conscientes quanto aos fatores de } \\
\text { risco de } \mathrm{DCV}^{\mathrm{a}} \text { (colesterol alto, estresse, } \\
\text { hipertensão, tabagismo obesidade, } \\
\text { diabetes, inatividade física e outros) do que } \\
\text { as estudantes. Comparações entre os } \\
\text { grupos de IMC revelaram que os } \\
\text { indivíduos obesos atribuíram menor } \\
\text { importância aos fatores de riscos de } \mathrm{DCV}^{\mathrm{a}}\end{array}$ & $\begin{array}{l}\text { As observações indicaram que os níveis de } \\
\text { consciência de fatores de risco de DCV precisam } \\
\text { ser aprimorados entre os estudantes } \\
\text { universitários. Portanto, é de extrema importância } \\
\text { que os profissionais de saúde da atenção primária } \\
\text { desenvolvam intervenções para a redução dos } \\
\text { níveis de risco de DCV }\end{array}$ \\
\hline 07 & $\begin{array}{l}\text { Em todos os nove países da ASEAN } \text {, }^{\mathrm{e}} \\
\text { houve prevalência de fatores de risco de } \\
\mathrm{DCV}^{\mathrm{a}} \text { entre os estudantes. Para os cinco } \\
\text { fatores de risco considerados (tabagismo, } \\
\text { dieta não saudável, obesidade, inatividade } \\
\text { física e uso nocivo de álcool), a } \\
\text { conscientização dos estudantes quanto ao } \\
\text { envolvimento desses fatores em DCV foi } \\
\text { baixa. Entretanto, as estudantes } \\
\text { apresentaram menor prevalência desses } \\
\text { fatores e uma crença "mais saudável" } \\
\text { quando comparado aos estudantes, exceto } \\
\text { na prática de atividade física }\end{array}$ & $\begin{array}{l}\text { A pesquisa constatou alta prevalência de fatores de } \\
\text { risco comportamentais de } \mathrm{DCV}^{\mathrm{a}} \text {, limitada } \\
\text { conscientização desses riscos e crenças de saúde } \\
\text { negativas. Os resultados podem favorecer o } \\
\text { desenvolvimento de estratégias de promoção da } \\
\text { saúde entre estudantes universitários da ASEAN }{ }^{\mathrm{e}} \text {. }\end{array}$ \\
\hline 08 & $\begin{array}{l}\text { O nível de conhecimento dos estudantes } \\
\text { quanto aos quatro indicadores de saúde } \\
\text { (colesterol, pressão arterial, glicemia e } \\
\text { IMC }^{d} \text { ) foi extremamente baixo. Entre os } \\
\text { estudantes de Farmácia do } 1^{\circ} \text { ano e } \\
\text { calouros, foram observadas diferenças em } \\
\text { termos de conhecimento sobre o colesterol } \\
\text { total e a pressão arterial. Em ambos os } \\
\text { grupos, menos de } 50 \% \text { dos estudantes } \\
\text { conheciam seu IMC }{ }^{\mathrm{d}} \text {, com um maior } \\
\text { percentual constatado entre aqueles do } 1^{\circ} \\
\text { ano profissional. A porcentagem de } \\
\text { respostas corretas para os outros três } \\
\text { indicadores de saúde (colesterol, glicemia e } \\
\text { IMC }^{\mathrm{d}} \text { ) foi baixa entre os estudantes }\end{array}$ & $\begin{array}{l}\text { A pesquisa revelou um reduzido nível de } \\
\text { conscientização de estudantes quanto aos seus } \\
\text { indicadores de saúde. Mostrou ainda que dois ou } \\
\text { mais anos de ensino superior e a escolha de uma } \\
\text { carreira profissional de saúde fazem a diferença no } \\
\text { nível de consciência dos estudantes quanto à saúde. } \\
\text { Todavia, mais pesquisas são necessárias para } \\
\text { entender o baixo nível de conscientização dos } \\
\text { indicadores críticos de saúde entre os estudantes }\end{array}$ \\
\hline 09 & $\begin{array}{l}\text { Embora insuficientes, a percepção e } \\
\text { conhecimento de alguns fatores de risco de } \\
\text { DCV }^{\text {a }} \text { (dislipidemia, hipertensão arterial e } \\
\text { alterações metabólicas) roram } \\
\text { significativamente melhores entre os } \\
\text { estudantes de graduação em relação aos }\end{array}$ & $\begin{array}{l}\text { Os resultados sugerem uma necessidade urgente de } \\
\text { conscientizar os estudantes do curso de Medicina } \\
\text { sobre a importância da obesidade e reduzida } \\
\text { atividade física no desenvolvimento de } \mathrm{DCV}^{\mathrm{a}}\end{array}$ \\
\hline
\end{tabular}




\begin{tabular}{|c|c|c|}
\hline & $\begin{array}{l}\text { calouros. Ao final do curso de Medicina, } \\
\text { muitos estudantes eram fumantes, apesar } \\
\text { de conhecerem os efeitos nocivos do } \\
\text { tabagismo. Eles apresentavam uma } \\
\text { reduzida consciência quanto à contribuição } \\
\text { da obesidade no desenvolvimento de DCV } \\
\end{array}$ & \\
\hline 10 & $\begin{array}{l}\text { Diante da possibilidade de desenvolver } \\
\text { DCV }^{\text {a }}, 85(53 \%) \text { estudantes mostraram-se } \\
\text { preocupados, embora apenas } 33(38 \%) \\
\text { tivessem adotado práticas preventivas } \\
\text { (atividade física e consumo regular de } \\
\text { gordura e sal). Do total de participantes, } \\
101 \text { (64\%) acreditavam que a vida na } \\
\text { escola de medicina tinha um efeito } \\
\text { prejudicial à saúde }\end{array}$ & $\begin{array}{l}\text { O estudo mostrou uma alta prevalência de } \\
\text { comportamentos de risco de } \mathrm{DCV}^{\mathrm{a}} \text { entre } \\
\text { estudantes do curso de Medicina }\end{array}$ \\
\hline 11 & $\begin{array}{l}\text { Os estudantes mais avançados no curso de } \\
\text { Medicina tinham maior conhecimento } \\
\text { sobre os fatores de risco de DVC } \\
\text { (colesterol, síndrome metabólica e terapia } \\
\text { hipolipemiante) do que aqueles que } \\
\text { estavam no início do curso. Apenas cerca } \\
\text { de } 20 \% \text { dos estudantes conheciam todos os } \\
\text { fatores de riscos incluídos na síndrome } \\
\text { metabólica. Fatores, como inatividade } \\
\text { física, obesidade, DM tipo } 2 \text {, tabagismo e } \\
\text { hipertensão, não foram consideravelmente } \\
\text { classificados como importantes condições } \\
\text { de risco de DCV }\end{array}$ & $\begin{array}{l}\text { O estudo mostrou claramente que o conhecimento } \\
\text { de estudantes do curso de Medicina sobre os fatores } \\
\text { de risco de } \mathrm{DCV}^{\mathrm{a}} \text { devem ser aprimorados. Ainda, a } \\
\text { educação médica deve fornecer conhecimentos } \\
\text { básicos que possibilitem a prevenção eficaz dessas } \\
\text { doenças }\end{array}$ \\
\hline 12 & $\begin{array}{l}\text { Em relação ao programa, o qual consistia } \\
\text { em aulas semanais, durante as quais eram } \\
\text { feitas atividades experimentais, } \\
\text { autoavaliações, discussões e trabalhos de } \\
\text { casa com estudantes afro-americanos, foi } \\
\text { constatada sua eficácia. Foram } \\
\text { identificados diversos benefícios da } \\
\text { intervenção, como aumento no } \\
\text { conhecimento sobre fatores de risco para } \\
\text { DCV e capacidade física, bem como } \\
\text { melhores hábitos alimentares e maior } \\
\text { conhecimento sobre as estratégias de } \\
\text { gerenciamento do estresse }\end{array}$ & $\begin{array}{l}\text { Oficinas experimentais com pequenos grupos } \\
\text { podem ser eficazes para a compreensão dos riscos e } \\
\text { estabelecimento de hábitos saudáveis para } \\
\text { prevenção de DCV }{ }^{\mathrm{a}} \text { entre os estudantes } \\
\text { universitários afro-americanos }\end{array}$ \\
\hline 13 & $\begin{array}{l}\text { O nível de conhecimento dos estudantes } \\
\text { sobre os fatores de risco de DCVa (IMC } \text {, } \\
\text { pressão arterial, glicose sérica, colesterol e } \\
\text { triglicerídeos) foi baixo. O percentual de } \\
\text { participantes com sobrepeso e obesidade } \\
\text { foi de } 18,8 \% \text {, para homens, e de } 22,1 \% \text {, } \\
\text { para mulheres. Observou-se uma relação } \\
\text { inversamente proporcional entre o maior } \\
\text { nível de conhecimento sobre os fatores de }\end{array}$ & $\begin{array}{l}\text { A pesquisa mostrou um conhecimento reduzido dos } \\
\text { fatores de risco de } \mathrm{DCV}^{\mathrm{a}} \text { entre estudantes do curso } \\
\text { de Enfermagem, além da presença desses fatores de } \\
\text { risco }\end{array}$ \\
\hline
\end{tabular}




\begin{tabular}{|c|c|c|}
\hline & $\begin{array}{l}\text { risco de } \mathrm{DCV}^{\mathrm{a}} \text { e a redução nos valores } \\
\text { séricos de glicose e colesterol }\end{array}$ & \\
\hline 14 & $\begin{array}{l}\text { Verificou-se que os estudantes do sexo } \\
\text { feminino tinham uma nutrição mais } \\
\text { adequada. Observou-se uma associação } \\
\text { positiva entre o conhecimento, a gravidade } \\
\text { percebida de DCV e autoeficácia com a } \\
\text { prática de atividade física, assim como } \\
\text { uma relação negativa entre esse } \\
\text { comportamento e as barreiras percebidas } \\
\text { (medo de estranhos, mau tempo e muitas } \\
\text { tarefas escolares) }\end{array}$ & $\begin{array}{l}\text { O estudo indicou que os contrastes entre as crenças } \\
\text { em saúde podem prever o comportamento de risco } \\
\text { de estudantes universitários frente a doenças } \\
\text { cardíacas. No entanto, são necessárias mais } \\
\text { pesquisas para verificar os preditores de } \\
\text { comportamentos de alto risco nesses estudantes }\end{array}$ \\
\hline 15 & 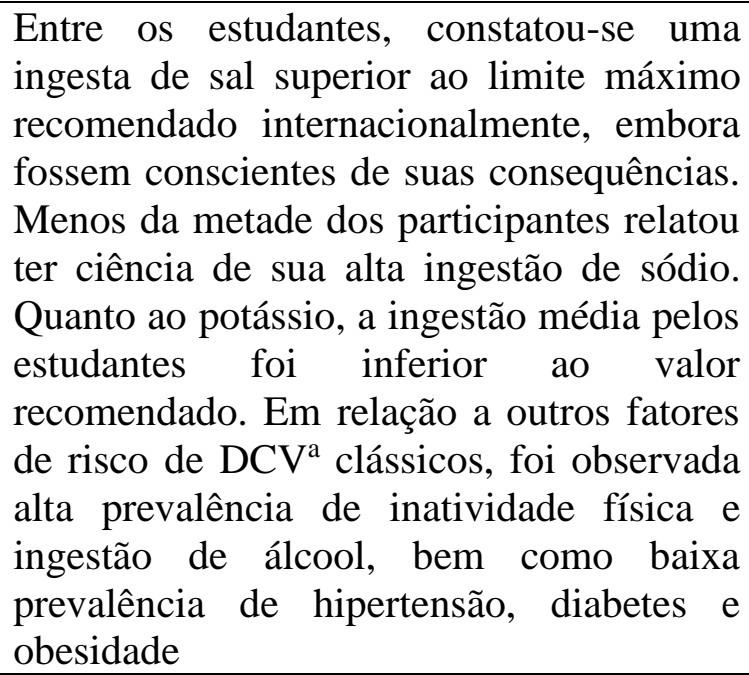 & $\begin{array}{l}\text { O estudo indicou uma alta ingestão de sal entre } \\
\text { estudantes do curso de Medicina e uma percepção } \\
\text { inadequada quanto ao seu nível de ingestão, bem } \\
\text { como atitude e comportamento insuficientes em } \\
\text { relação ao seu controle. Diante desse cenário, faz- } \\
\text { se necessária a educação nutricional para aprimorar } \\
\text { os conhecimentos e hábitos alimentares desses } \\
\text { estudantes }\end{array}$ \\
\hline 16 & $\begin{array}{l}\text { Entre os estudantes, foram constatados } \\
\text { baixos níveis de conhecimento sobre } \\
\text { fatores de risco de DCV }{ }^{\mathrm{a}} \text { (tabagismo, } \\
\text { álcool, dieta não saudável, inatividade } \\
\text { física, IMC }{ }^{\mathrm{d}} \text { e estresse). Dos participantes, } \\
46,1 \% \text { eram fumantes, } 31,9 \% \text { consumiam } \\
\text { álcool, 20,8\% tinham sobrepeso, } 1,6 \% \\
\text { eram obesos e 26,1\% tinham doenças } \\
\text { crônicas. Os resultados mostraram que os } \\
\text { estudantes não tinham comportamentos } \\
\text { saudáveis, como prática de exercício físico, } \\
\text { nutrição adequada e controle do estresse }\end{array}$ & $\begin{array}{l}\text { As avaliações dos níveis de conhecimento } \\
\text { mostraram que os estudantes não possuíam } \\
\text { conhecimento adequado e que existia uma relação } \\
\text { positiva e significativa entre comportamentos } \\
\text { positivos de saúde e níveis de conhecimento sobre } \\
\text { fatores de riscos de DCV }{ }^{\text {a }} \text {. Nesse sentido, pode-se } \\
\text { sugerir que estudos e triagem em doenças crônicas } \\
\text { e consultas médicas regulares podem prevenir essas } \\
\text { doenças }\end{array}$ \\
\hline 17 & $\begin{array}{l}\text { Os participantes conheciam os fatores de } \\
\text { risco de DCV (diabetes, sobrepeso/ } \\
\text { obesidade, hipertensão, hiperlipidemia, } \\
\text { tabagismo), mas não se viam em risco de } \\
\text { desenvolvê-las. Quando avaliados os } \\
\text { fatores de riscos de DCV } \text {, constatou-se } \\
\text { que mais de } 50 \% \text { dos estudantes tinham } \\
\text { um ou mais desses fatores }\end{array}$ & $\begin{array}{l}\text { O alto nível de conhecimento dos fatores de risco } \\
\text { de } \mathrm{DCV}^{\mathrm{a}} \text { não foi suficiente para reduzir os riscos } \\
\text { dessas doenças entre os estudantes. Dessa forma, } \\
\text { alterar a percepção desses fatores pode } \\
\text { desempenhar um papel maior na redução dos riscos } \\
\text { dessas doenças a longo prazo }\end{array}$ \\
\hline
\end{tabular}

${ }^{\mathrm{a} D o e n c ̧ a s ~ C a r d i o v a s c u l a r e s ; ~}{ }^{\mathrm{b}}$ Hipertensão Arterial Sistêmica; ${ }^{\mathrm{c}}$ Diabetes Mellitus; ${ }^{\mathrm{d}}$ Índice de Massa Corporal; ${ }^{\mathrm{e} A s s o c i a c ̧ a ̃ o ~ d a s ~ N a c ̧ o ̃ e s ~ d o ~ S u d e s t e ~ A s i a ́ t i c o . ~}$

Fonte: Os autores 


\section{DISCUSSÃO}

As estatísticas apresentadas pela literatura Editora Elsevier ${ }^{35}$. Quanto ao idioma dos artigos revelam que as DCV representam uma das aqui inseridos, o fato de todos estarem em língua principais causas de morte no contexto mundial. Em inglesa pode ser justificado por ser ela uma das vista disso, a relevância de estudar os fatores de estratégias aplicadas para a internacionalização risco cardiovasculares em universitários se baseia científica ${ }^{36}$ ou pelo fato de possíveis bases de dados na conjuntura de que esse público, pelas que incluem artigos no idioma português não terem transformações físicas, emocionais e sociais, busca sido incluídos na revisão.

pela independência e vivência de novas

No que se refere ao maior número de estudos experiências, associadas a uma elevada carga de publicados em diferentes anos, esse resultado pode estresse proporcionada pelo ambiente universitário, sugerir a relevância e interesse da temática aqui torna-se vulnerável a comportamentos de risco, retratada em distintos momentos do tempo. Quanto mesmo diante do acesso a informações ${ }^{19}$.

ao local da realização dos estudos incluídos nessa

Assim, compreender esses fatores pode revisão, o destaque para a Turquia, além dos EUA, prevenir, manter ou restabelecer a saúde dessa foi um dado inesperado, já que a China tem se população e evitar impactos socioeconômicos ${ }^{33}$, destacado pelo incentivo ao desenvolvimento de visto que esses elementos podem ser modificáveis pesquisas ${ }^{37}$. O destaque dos EUA pode ser de acordo com o ambiente e tempo de exposição ${ }^{34}$. justificado pela possibilidade desse país

$\mathrm{Na}$ presente revisão, foram avaliadas as desenvolver mais pesquisas nessa área como produções que abordaram a prevalência, consequência dos hábitos alimentares, estilo de vida conhecimento, atitudes e práticas em relação aos e acometimento da população por doenças fatores de risco de DCV em estudantes cardiovasculares, uma vez que é considerado como universitários. A constatação desses aspectos em um dos líderes globais em ciência e tecnologia ${ }^{38}$. adultos jovens possibilita identificar o nível de Sobre a área de publicação dos artigos, o suscetibilidade desses indivíduos a essas doenças. grande quantitativo de trabalhos disponibilizados Portanto, a compilação dos estudos ajudará a em periódicos da área médica, embora evidencie a identificar as lacunas no conhecimento, o que importância da temática para a Medicina, sugere poderá melhorar as práticas e políticas direcionadas uma deficiência na integração entre as diferentes à promoção e prevenção das DCV entre os áreas da saúde, o que vai de encontro ao ditado pela universitários. literatura. De acordo com os autores ${ }^{39}$, o trabalho

Quando avaliado o quantitativo de trabalhos em saúde deve envolver ações interdisciplinares, incluídos nessa revisão, de acordo com a base de multidisciplinares e transdisciplinares. dados, o destaque para o Scopus pode ser concebido Com relação à grande parte das publicações por ser ele a maior base de dados, contendo apresentarem, como desenho metodológico, o https://doi.org/10.31011/reaid-2021-v.95-n.36-art.1122 Rev Enferm Atual In Derme v. 95, n. 36, 2021 e-021136 
estudo descritivo, embora descreva dados que foram

No que diz respeito aos resultados verificados, registrados, analisados, classificados e apresentados pelas pesquisas incluídas na revisão, o interpretados, sem a interferência do pesquisador e fato de que, em geral, os estudantes apresentaram com uso de técnicas padronizadas de coleta de baixos níveis de conhecimento sobre os fatores de $\operatorname{dados}^{40}$, não busca avaliar uma possível associação risco de DCV, foi surpreendente, especialmente se entre a causa da doença e seu efeito ${ }^{41}$. Sobre o considerado que esse fenômeno ocorreu entre predomínio de estudos com nível VI de evidência, estudantes de diferentes faculdades e tempo de esse achado sugere que as publicações não curso. Ressaltando ainda o imprevisto desse achado, apresentam fortes evidências, o que pode prejudicar sabe-se que esses estudantes apresentavam sua aplicabilidade clínica ${ }^{32}$. inúmeros comportamentos de risco para essas

Quando analisados os objetivos dos artigos doenças e que a Universidade representa um incluídos, a maior quantidade de publicações que importante meio de disseminação das informações avaliaram os conhecimentos, atitudes e práticas em sobre esses fatores de risco a muitos jovens ${ }^{43}$. relação aos fatores de risco de DCV de estudantes Contudo, esse dado pode resultar da forma universitários superou as expectativas dessa revisão como a mídia e o consumismo da sociedade atual por não terem se limitado ao conhecimento desses vinculam esses fatores de risco ao prazer, fatores. Esse resultado é importante no sentido de especialmente em propagandas de fast food, além que intervenções voltadas à promoção e prevenção de que se admite que a atuação dos fatores de risco de DCV, de cunho individual ou coletivo, são comportamentais sobre a saúde é geralmente melhores efetuadas se embasadas no conhecimento manifestada em idades mais avançadas ${ }^{44}$. Esse dado das atitudes e práticas do público a que se também pode decorrer do nível de escolaridade dos destinam $^{42}$. pais, especialmente da mãe, pelo papel que essa

O mesmo pode ser dito em relação aos assume no cuidado com os filhos ${ }^{45}$. Realmente, em estudos aqui retratados, os quais se propuseram a pesquisa dos autores ${ }^{46}$, os autores observaram que apresentar a prevalência de fatores de risco ou da os estudantes, cujas mães tinham 5 a 11 anos de própria DCV entre os universitários, os resultados estudo, apresentaram maior possibilidade de ter um da avaliação desse risco e a análise do impacto da fator de risco a mais em comparação àqueles, cujas educação sobre o conhecimento e reconhecimento mães tinham escolaridade maior ou igual a 12 anos. da importância da implementação de medidas Dessa forma, independentemente da causa da preventivas contra DCV. Realmente, conhecer esses desinformação, a sua consequência pode não diferentes aspectos pode interferir nas ações diminuir a incidência de DVC entre os estudantes destinadas à prevenção, manutenção e universitários, além de favorecer a elevação dos restabelecimento da saúde de universitários diante custos para os setores públicos e privados ${ }^{47}$. dessas doenças. 
No que se refere aos fatores de risco de DCV, aos fatores de risco de $\mathrm{DCV}$, os estudantes não a literatura classifica-os em fatores de riscos acreditavam em sua vulnerabilidade a essas modificáveis (ambientais e comportamentais), como doenças, o que pode ser compreendido se admitido o tabagismo, alcoolismo, colesterol sérico elevado, que a idade acima de 45 anos para homens e 55 hipertensão arterial sistêmica, inatividade física, anos para mulheres é tida como um fator de estresse e condições secundárias (DM, obesidade, susceptibilidade a $\mathrm{DCV}^{52}$. Para a não adoção de uso de anticoncepcional e obesidade abdominal), e medidas preventivas pelos estudantes, esse fatores de risco não modificáveis (genéticos e resultado pode se associar à própria crença de que biológicos), representados pela hereditariedade, não são susceptíveis a DCV e/ou falta de tempo ${ }^{53}$. sexo e idade avançada ${ }^{44}$.

Quanto à ausência de prática de um estilo saudável,

Especificamente, os fatores de risco de DCV apesar da consciência quanto aos fatores de risco, relatados nos estudos aqui incluídos foram ela pode ser explicada por problemas na inúmeros e compreenderam tanto os modificáveis administração do tempo, necessidade de obter um quanto os não modificáveis, com um quantitativo bom desempenho ${ }^{53}$, comprometimento dos hábitos maior entre os primeiros. Esses fatores foram alimentares e horários de refeições e adoção de um também observados por outros autores ${ }^{44,48}$, os quais estilo de vida mais sedentário ${ }^{54}$.

destacaram o sedentarismo. Esse, conjuntamente

Em relação ao baixo nível de conhecimento com a hipertensão arterial, tabagismo, hiperglicemia sobre os indicadores de saúde entre os e sobrepeso/obesidade, figura como um dos fatores universitários, esse achado é preocupante por de risco de DCV globalmente conhecidos ${ }^{49}$.

sugerir que esses estudantes desconhecem a

Nessa revisão, o tabagismo foi o fator de risco realidade vivenciada por eles, o que pode modificável mais citado pelos estudantes, o que comprometer a aplicação de políticas e estratégias pode estar associado à elevada prática desse hábito voltadas a uma boa atuação e desempenho do entre os universitários ${ }^{50}$ e/ou ao fato de ser ele um sistema de saúde, bem como do monitoramento dos principais fatores de risco cardiovascular dessas doenças nessa população.

modificável ${ }^{51}$. Segundo a literatura, o tabagismo é $\quad$ No que se refere à percepção dos estudantes responsável por inúmeras e diversas doenças universitários de que o curso de Medicina era crônicas e por metade de todas as mortes prejudicial à saúde, esse resultado corrobora com os evitáveis ${ }^{50}$. A ele é atribuída a elevação do estresse autores ${ }^{55}$, os quais relataram que o desafio das oxidativo, do processo inflamatório e da disfunção escolas de Medicina é proporcionar ambientes endotelial e plaquetária, fenômenos que podem acadêmicos que não comprometam a saúde e causar dano ao organismo ${ }^{51}$. estimulem o progresso nos estudos.

No tocante ao resultado apresentado pelos Especificamente, o estresse crônico vivenciado por artigos de que, apesar de terem a consciência quanto esses estudantes pode aumentar o risco de doenças https://doi.org/10.31011/reaid-2021-v.95-n.36-art.1122 Rev Enferm Atual In Derme v. 95, n. 36, 2021 e-021136 
cardiometabólicas e neurodegenerativas, além de adoção de medidas preventivas e de estilo de vida causar declínio cognitivo e efeitos adversos no saudável, ressalta a urgência da elaboração e hipocampo ${ }^{55}$. aplicação de estratégias voltadas à promoção da

Esse dado pode ser ainda entendido se saúde.

admitido que estudantes de Medicina apresentam, em geral, um estilo de vida inadequado marcado pelo sedentarismo, hábitos alimentares inadequados, limitado tempo para o lazer e alta internacional sobre a temática aqui retratada. Nesse carga de trabalho, assim como elevada frequência âmbito, poucos foram os estudos brasileiros de realização de provas e expectativa de si mesmos, incluídos, o que desperta para a necessidade de isolamento social, competição com amigos e condução de mais pesquisas relacionadas ao excesso de conteúdo a ser estudado ${ }^{56,57}$. conhecimento dos fatores de risco de doenças

Com respeito às diferenças entre os sexos cardiovasculares entre os estudantes universitários, quanto às atitudes e práticas em saúde dos proporcionando a elaboração de novas revisões da estudantes universitários observados pelas literatura e fortalecendo a prática baseada em pesquisas aqui abordadas, esse resultado parece evidências.

refletir os estereótipos relacionados ao sexo, especialmente expressando a imagem do homem como aquele que é mais forte, que não cuida de si nem do outro e que não busca os serviços de saúde. Em contrapartida, a mulher é aquela que é naturalmente cuidadora e que requer mais atenção pelas particularidades do organismo feminino ${ }^{58}$. Esse dado é preocupante, já que os homens parecem ser mais vulneráveis a enfermidades crônicas e 2 . graves, além de terem uma morte mais precoce ${ }^{59,60}$.

\section{CONCLUSÕES}

Pode-se concluir que o reduzido conhecimento sobre os fatores de risco de doenças cardiovasculares apresentado por estudantes universitários de diferentes instituições de ensino e tempo de curso, associado ao não reconhecimento de vulnerabilidade a essas enfermidades e não

\section{REFERÊNCIAS}

1. Bao TQ, Hoang VM, Lan VH, Linh BP, Giang $\mathrm{KB}$, Nga PQ, et al. Risk factors for NonCommunicable Diseases among adults in Vietnam: Findings from the Vietnam STEPS Survey 2015. J Glob Heal Sci. Korean Society of Global Health; 2020;2(1). DOI: 10.35500/jghs.2020.2.e7

WHO. Who Global Status Report on NonCommunicable Diseases 2014. [En ligne]. WHO World Health Organization; 2014 [cité le 7 janvier 2021].

3. Malta DC, Andrade SC, Claro RM, Bernal RTI, Monteiro CA. Evolução anual da prevalência de excesso de peso e obesidade em adultos nas capitais dos 26 estados brasileiros e no Distrito Federal entre 2006 e 2012. Rev Bras Epidemiol. 2014;17(SUPPL. 1):267-76. DOI: $\quad 10.1590 / 1809$ 4503201400050021

\section{WORLD HEALTH ORGANIZATION.}


Noncommunicable diseases. Key Facts; 2018. WHO 2018.

5. Figueiredo AR, Cabral ABS, Almeida ACO, Ribeiro MA, Araújo MR de S, Ferreira MFDC, et al. Ação educativa acerca dos fatores de riscos de doenças cardiovasculares em adolescentes: relato de experiência. Rev Eletrônica Acervo Saúde. 2020;(42):e2292. DOI: $10.25248 /$ reas.e2292.2020

6. Zainel AJAL, Nuaimi AS Al, Mohamed Ahmed Syed, A/Qotba HA. Risk Factors Associated with Cardiovascular Diseases among Adults Attending the Primary HealthCare Centers in Qatar, a CrossSectional Study. J Community Med Public Heal [En ligne]. 2020 [cité le 6 janvier 2021];4.

Disponible: https://www.gavinpublishers.com/articles/res earch-article/Journal-of-CommunityMedicine-Public-Health/risk-factorsassociated-with-cardiovascular-diseasesamong-adults-attending-the-primary-healthcare-centers-in-qatar-a-cross-sectional-study

7. Bhupathi V, Mazariegos M, Cruz Rodriguez JB, Deoker A. Dairy Intake and Risk of Cardiovascular Disease. Vol. 22, Current Cardiology Reports Springer; 2020.

8. Précoma DB, de Oliveira GMM. Atualização da Diretriz de Prevenção Cardiovascular da Sociedade Brasileira de Cardiologia. Soc Bras Cardiol [En ligne]. 2019 [cité le 10 janvier 2021];113(4):787-891. Disponible: https://www.scielo.br/scielo.php?pid=S0066782X2019001000787\&script=sci_arttext\&tln $\mathrm{g}=\mathrm{pt}$

9. A.F. S, D.B. P, J.P. A, H CF, J.F.K. S, G.M.M. O. I Diretriz Brasileira de Prevenção Cardiovascular. Arq Bras Cardiol [En ligne]. 2013 [cité le 10 janvier 2021];101(6). Disponible:

https://www.scielo.br/scielo.php?pid=S0066782X2013004500001\&script=sci_arttext\&tln $\mathrm{g}=\mathrm{pt}$

10. Martins LN, Souza LS de, Silva CF da, Machado RS, Silva CEF da, Vilagra MM, et al. Prevalência dos Fatores de Risco Cardiovascular em Adultos Admitidos na Unidade de Dor Torácica em Vassouras, RJ. onlineijcs.org [En ligne]. 2011 [cité le 10 janvier 2021];24(5):299-307. Disponible: http://www.onlineijcs.org/english/sumario/24 /pdf/v24n5a05.pdf

11. Akbari G. Molecular mechanisms underlying gallic acid effects against cardiovascular diseases: An update review. Avicenna J Phytomedicine. 2020;10(1):11-23. DOI: 10.22038/ajp.2019.13537

12. Isbister J, Semsarian C. Cardiovascular genomics and sudden cardiac death in the young. Aust J Gen Pract. 2019;48(3):90-5. DOI: 10.31128/AJGP-09-18-4715

13. Herdy A, López-Jiménez F, ... CT-AB, 2014 U. Diretriz sul-americana de prevenção e reabilitação cardiovascular. ArqBrasCardiol [En ligne]. 2014 [cité le 6 janvier 2021];103(2):1-31.

Disponible: https://www.scielo.br/scielo.php?pid=S0066782X2014003000001\&script=sci_arttext\&tln $\mathrm{g}=\mathrm{pt}$

14. Spence JD, Pilote L. Importance of sex and gender in atherosclerosis and cardiovascular disease. Vol. 241, Atherosclerosis 2015. p. 208-10.

15. Back IR, Dias BC, Batista VC, Ruiz AGB, Peruzzo HE, Druciak CDA, et al. $\langle\mathrm{b}\rangle$ Fatores de risco para doenças cardiovasculares em universitários: diferenças entre os sexos / Risk factors for cardiovascular diseases in university students: differences between the sexes $\langle\mathrm{b}\rangle$. Ciência, Cuid e Saúde. $2019 ; 18(1)$. DOI:

10.4025/cienccuidsaude.v18i1.40096

16. Oliveira CQ de. AVALIAÇÃO DO SOBREPESO, OBESIDADE, ALTERAÇÕES DA PRESSÃO ARTERIAL E DA APTIDÃO FÍSICA ENTRE ADOLESCENTES DA REDE MUNICIPAL DE ENSINO DE PETRÓPOLIS. PróReitoria Pesqui e Pós-Graduação [En ligne]. 2020 [cité le 10 janvier 2021];0(0). 
Disponible:

http://seer.ucp.br/seer/index.php/propep/articl e/view/1693

17. Cotten E, Prapavessis H. Increasing Nonsedentary Behaviors in University Students Using Text Messages: Randomized Controlled Trial. JMIR mHealth uHealth. 24. 2016;4(3):e99. DOI: 10.2196/mhealth.5411

18. Mussi FC, Pitanga FJG, Pires CG da S. Tempo sentado acumulado como discriminador de sobrepeso, obesidade, obesidade abdominal e alterações lipídicas em universitárias de enfermagem. Rev Bras Cineantropometria e Desempenho Hum. Universidade Federal de Santa Catarina; 2017;19(1):40-9. DOI: 10.5007/19800037.2017v19n1p40

19. Silva LDC, Costa JCM, Nunes FDO, Azevedo PR. Health risk behaviors in college students of a public institution. Rev Pesqui 26. Cuid é Fundam Online. 2020;12:544-50. DOI: $10.9789 / 2175-5361 . r p c f o . v 12.8635$

20. Lima MMM, Felício JF, M'batna AJ, Ferreira DS, Cavalcante MCSL, Dutra FCS, et al. Perfil de manifestação de estresse em universitários: um estudo transversal. Rev Enferm Atual In Derme. 2021;95(33):1-14. 27. DOI: 10.31011/reaid-2021-v.95-n.33-art.903

21. Sogari G, Velez-Argumedo C, Gómez MI, Mora C. College students and eating habits: A study using an ecological model for healthy behavior. 2018;10(12):1-16.

Nutrients. $10.3390 /$ nu 10121823

22. Calixto L de F, Montiel EM da S, Rocha KS dos S, Lima D von G, Marcon MV, Kluthcovsky ACGC, et al. Fatores de risco para doenças cardiovasculares em estudantes de medicina. Int J Dev Res. 29. 2019;9(10):30725-31. DOI: 10.1590/S0066782X2005001400011

23. Macedo TTS de, Mussi FC, Geovana C, Ramon J, Teixeira B, Portela PP. Perfil alimentar , clínico e padrão de atividade física em ingressantes universitários de enfermagem. Rev Cubana Enferm [En ligne]. 2019 [cité le 8 octobre 2020];1(25):2-21. Disponible:

http://www.revenfermeria.sld.cu/index.php/e nf/article/view/1785

Mendes MLM, Silva FR da, Messia CMB de O, Carvalho PGS de, Silva TFA da. Hábitos alimentares e atividade física de universitários da área de saúde do município de Petrolina-PE. Tempus Actas de Saúde Coletiva. 2016;10(2):205. DOI: 10.18569/tempus.v10i2.1669

25. Pires CG da S, Mussi FC, Cerqueira BB de, Pitanga FJG, Silva DO da. Prática de atividade física entre estudantes de graduação em enfermagem. Acta Paul Enferm. 2013;26(5):436-43. DOI: 10.1590/s010321002013000500006

Miranda MLP, Kamiji MM, Torezzan C, Antunes AEC. Avaliação antropométrica e análise do consumo alimentar de universitários da Faculdade de Ciências Aplicadas - UNICAMP. Segurança Aliment e Nutr. 2015;21(2):461. DOI: 10.20396/san.v21i2.8634475

Rezende LCM, Fontes WD de, Martins KP, Santos SR. Fatores de risco para hipertensão arterial sistêmica em crianças. Rev enferm UFPE line, Recife,. 2014;8(8):2611-6. DOI: 10.5205/reuol.6081-52328-1-

SM.0808201405

Souza MT de, Silva MD da, Carvalho R de. Revisão integrativa: o que é e como fazer. einstein [En ligne]. 2010 [cité le 10 janvier 2021];8(1):102-6. Disponible: https://www.scielo.br/pdf/eins/v8n1/pt_16794508-eins-8-1-0102

Sousa LMM de, Marques-Vieira CMA, Severino SSP, Antunes AV. A metodolgia de revisão integrativa da literatura em enfermagem. Rev Investig EM Enferm [En ligne]. 2017;17-26. Disponible: http://www.sinaisvitais.pt/images/stories/Rie/ RIE21.pdf\#page $=17$ 
30. Ursi ES, Gavão CM. Prevenção de lesões de pele no perioperatório: revisão integrativa da literatura. Rev Lat Am Enfermagem. 2006;14(1):124-31. DOI: 10.1590/s010411692006000100017

31. Ferreira MJA da S, Lima RF, Albuquerque 37. AJ, Santos VEFA, Silva ARS, Medeiros CSQ. O Cuidado à Criança Com Insuficiência Renal: Uma Revisão Integrativa da Literatura. Cad Grad - ciências Biológicas e da Saúde FACIPE [En ligne]. 2013 [cité le 10 janvier 2021];1(1):37-49. Disponible: http://periodicos.set.edu.br/facipesaude/articl e/view/1058

32. Stillwell SB, Fineout-Overholt E, Melnyk BM, Williamson KM. Evidence-based practice, step by step: Searching for the evidence. Am J Nurs. Am J Nurs; 2010;110(5):41-7. DOI: 10.1097/01.NAJ.0000372071.24134.7e

33. Aprígio D de P, Araújo VB de, Omatsu VL, Mendes BM, Jesus JL de, Furtado O, et al. Evaluation of risk factors for the development of cardiovascular diseases in students of Serra dos Órgãos University. Rev DA JOPIC [En ligne]. 2020 [cité le 10 janvier 2021];3(6):15-22. Disponible: http://www.revista.unifeso.edu.br/index.php/j opic/article/view/1901

34. Gasparotto G da S, Gasparotto LPR, Salles MR de, Campos W de. Fatores de risco cardiovascular em universitários: comparação entre se- xos, períodos de graduação e áreas de estudo. Med (Ribeirão Preto) [En ligne]. 2013 [cité le 10 janvier 2021];46(2):154-63.

Disponible:

http://revista.fmrp.usp.br/

35. ALBACH, VM; MEDEIRO M. Utilização da Revisão Bibliográfica Sistemática em Turismo: Panorama Internacional e Iberoamericano dos Trabalhos Presentes no Scopus e Redalyc. Publ UEPG Appl Soc Sci, Ponta Grossa. 2020;28(e 202014492):1-14.

36. Cintra PR, Silva MDP da, Furnival AC. Uso do inglês como estratégia de internacionalização da produção científica em Ciências Sociais Aplicadas: estudo de caso na SciELO Brasil. Em Questão. 2020;26(1):17-41. DOI: 10.19132/1808$5245261.17-41$

Oliveira GX de, Vestena D, Costa CRR da, Traverso LD, Bichueti RS. Internacionalização das universidades: estudo sobre a produção científica. Rev Gestão e Desenvolv. 2020;17(1):196. DOI: 10.25112/rgd.v17i1.2020

38. Lymn N, Bates S. State of US science enterprise report shows US leads in $\mathrm{S} \& \mathrm{E}$ as China rapidly advances. Natl Sci Found [En ligne]. 2018 [cité le 6 janvier 2021];1-5. Disponible:

https://www.nsf.gov/news/news_summ.jsp?o rg=NSF\&cntn_id=244271\&preview=false

39. Guimarães BE de B, Castelo Branco AB de A. Trabalho em Equipe na Atenção Básica à Saúde: Pesquisa Bibliográfica. Rev Psicol e Saúde. 2020;12(1):143-55. DOI: $10.20435 /$ pssa.v12i1.669

40. Franco FR, Rocha RT, Pereira CA. Humanidades \& Tecnologia em Revista (FINOM)- a importância de desenvolvimento do Centro de Atenção à Saúde do Idoso em Paracatu/MG: a busca por uma assistência adequada e o papel do enfermeiro. Humanidades Eecnologia em Rev [En ligne]. 2020 [cité le 6 janvier 2021];23. Disponible: http://revistas.icesp.br/index.php/FINOM_Hu manidade_Tecnologia/article/view/1210

41. Oliveira MA, Vellarde GC, Sá RAM de. Entendendo a pesquisa clínica III: estudos de coorte. Femina [En ligne]. 2015 [cité le 6 janvier 2021];43(34):105-10. Disponible: https://pesquisa.bvsalud.org/portal/resource/p t/lil-763819?lang=en

42. Denti IA, Brock F, Biasi LS De. Comportamento e atitudes para prevenir doenças cardiovasculares. Perspect Erechim [En ligne]. 2016 [cité le 10 janvier 2021];40(149):139-51. Disponible: http://www.uricer.edu.br/site/pdfs/perspectiv 
a/149_554.pdf

43. Abdela OA, Ayalew MB, Yesuf JS, Getnet SA, Biyazin AA, Lemma MY, et al. 49. Ethiopian university students' knowledge and perception towards cardiovascular disease risk factors: a cross sectional study. Am J Cardiovasc Dis [En ligne]. 2019 [cité le 6 janvier 2021];9(1):1-7. Disponible: https://www.ncbi.nlm.nih.gov/pmc/articles/P MC6420697/

44. Toledo RA, Jurca CF, Rios CAG, Lima LFG, Rodrigues VO, Santos RO. Fatores de risco cardiovascular modificáveis em estudantes de medicina de um centro universitário brasileiro. Brazilian J Dev. 2019;5(10):19944-57.

10.34117/bjdv5n10-197

DOI:

45. Lazzarini A, Sampaio C, ... VG-RC em, 2018 U. Mulheres na Ciência: papel da educação sem desigualdade de gênero. Rev Ciência em Extensão [En ligne]. 2018 [cité le 6 janvier 2021];14(2):188-94. Disponible: https://ojs.unesp.br/index.php/revista_proex/a rticle/view/1717

46. Paulitsch RG, Dumith SC, Susin LRO. Simultaneidade de fatores de risco comportamentais para doença cardiovascular em estudantes universitários. Rev Bras Epidemiol. 2017;20(4):624-35. DOI: 10.1590/1980-5497201700040006

47. Dos Santos CT, Raul Ferreira de Souza Machado, Thais Lemos de Souza Macêdo, Dandhara Martins Rebello, João Paulo Brum 53. Paes, Ivana Picone Borges de Aragão. Rastreamento do autoconhecimento e da presença dos fatores de risco cardiovascular em população da periferia da cidade de Vassouras: Dados preliminares. Rev Saúde. 2019;10(2):12-7.

DOI:

10.21727/rs.v10i2.1882

48. Mascena GV, Cavalcante MSB, Marcelino GB, Holanda SA, Brandt CT. Fatores de risco cardiovascular em estudantes da Faculdade de Ciências Médicas de Campina Grande. Med (Ribeirao Preto Online).
2012;45(3):322. DOI: 10.11606/issn.21767262.v45i3p322-328

Teston EF, Cecilio HPM, Santos AL, De Arruda GO, Radovanovic CAT, Marcon SS. Factors associated with cardiovascular diseases in adults. Med. 2016;49(2):95-102. DOI: $\quad 10.11606 /$ issn.2176-7262.v49i2p95102

50. Macêdo TTS de, Mussi FC, Palmeira CS, Mendes AS. Consumo de bebida alcoólica, tabaco e drogas ilícitas em ingressantes universitários da área de enfermagem. Rev Divulg Científica Sena Aires. 2020;9(1):77-88

DOI:

10.36239/revisa.v9.n1.p77a88

51. Jacondino CB, Schwanke CHA, Closs VE, Gomes I, Borges CA, Gottlieb MGV. Associação do tabagismo com biomarcadores REDOX e fatores de risco cardiometabólicos em idosos. Cad Saúde Coletiva. 2019;27(1):45-52. DOI: 10.1590/1414$462 \times 201900010279$

52. Freire AK da S, Alves NCC, Santiago EJP, Tavares AS, Teixeira D da S, Carvalho IA, et al. Panorama No Brasil Das Doenças Cardiovasculares Dos Últimos Quatorze Anos Na Perspectiva Da Promoção À Saúde. Rev Saúde e Desenvolv [En ligne]. 2017 [cité le 6 janvier 2021];11(9):21-44. Disponible:

https://www.uninter.com/revistasaude/index. $\mathrm{php} / \mathrm{saudeDesenvolvimento/article/view/776}$

Dias AC, Carlotto R, de Oliveira C, Teixeira M. Dificuldades percebidas na transição para a universidade. Rev Bras Orientação Prof. Associacao Brasileira de Orientadores Profissionais; 2019;20(1):19-30. DOI: 10.26707/1984-7270/2019v20n1p19

54. Prati SRA, Porto WJ, Ferreira L. Estilo de vida de universitários: uma investigação sobre hábitos alimentares, atividade física e estresse. BIOMOTRIZ. 2020;14(2):69-78. DOI: 10.33053/biomotriz.v14i2.30

55. Stegers-Jager KM, Savas M, van der Waal J, 
van Rossum EFC, Woltman AM. Gender- 59. specific effects of raising Year-1 standards on medical students' academic performance and stress levels. Med Educ. Blackwell Publishing Ltd; 2020;54(6):538-46. DOI: 10.1111/medu.14068

56. Steiner-Hofbauer V, Holzinger A. How to Cope with the Challenges of Medical 60. Education? Stress, Depression, and Coping in Undergraduate Medical Students. Acad Psychiatry. 2020;44(4):380-7. DOI: 10.1007/s40596-020-01193-1

57. Thomas E, M G. Prevalence and Determinants of Overweight and Obesity among Medical Students. Nsatl J Physiol Pharm Pharmacol. 2020;10(1):1. DOI: 10.5455/njppp.2020.10.1035506112019

58. Bertolini DNP, Simonetti JP. The male genre and health care: the experience of men at a health center. Esc Anna Nery - Rev Enferm. 2014;18(4):722-7. DOI: $10.5935 / 1414-$ 8145.20140103

Submissão: 2021-05-25

Aprovado: 2021-09-26
Balbino CM, Silvino ZR, Santos JS dos, Joaquim FL, Souza CJ de, Santos LM dos, et al. Os motivos que impedem a adesão masculina aos programas de atenção a saúde do homem. Res Soc Dev. 2020;9(7):e389974230. DOI: 10.33448/rsdv9i7.4230

60. Bruno PS, Zanini MTB, Soratto MT, Feltrin JO. Saúde do homem: limites e possibilidades. Enferm Bras [En ligne]. 2011 [cité le 10 janvier 2021];10(5):274-9. Disponible:

http://www.portalatlanticaeditora.com.br/ind ex.php/enfermagembrasil/article/view/3874

\section{Autor correspondente}

Ana Caroline Rocha de Melo Leite, Universidade da Integração Internacional da Lusofonia Afro-Brasiliera - Campus das Auroras - Rua José Franco de Oliveira, s/n, CEP - 62.790-970 - Redenção-Ceará, fone (85) 99168-0679 e e-mail: acarolmelo@unilab.edu.br 